\title{
The Model of a Person Taking a Bath with Hot Water
}

\author{
Danyang $\mathrm{Li}^{1, \mathrm{a}^{*}}$ \\ ${ }^{1}$ School of Environmental Science and Engineering Department, North China Electric Power \\ University, Baoding 071003, China \\ a522458896@qq.com
}

Keywords: Model; Bathtub; Hot water; A person.

\begin{abstract}
In order to create a comfortable and cost-effective hot bath condition for everyone, we invent a bathroom environment to simulate the process of people taking a bath. Then, what are a hot bath made of There must be a bath crock, a tired man who is eager to clean himself. In this way, we got the two key elements of a bath. Then we can find a really good bath strategy to achieve a comfortable bath by coordinating the two factors. We put aside the man who wants to take a bath. And we just consider the heat transfer inside the bathtub which filled with hot water in a constant temperature bathroom. We divided the water in the bathtub into many small water cube. Then we simulate the change of the temperature of every water cube from outside to inside. According to those changeable water cubes, we build a temperature field of the hot water in the bathtub in time and space.
\end{abstract}

\section{Introduction}

If you want to talk about all the study of hot water, it's as if the keeping in good health. Then we launched these things through to all kinds of associations of the bathroom. A cup of hot water is placed on the table. It grows cool slowly. We think constant temperature tank of tropical fish. The lake which is injected hot water, the loss of the temperature of stationary liquid and disorders fluid, heat exchange between the liquid and gas and solid. In general, it is the change of temperature field.

Currently, it has the shadow of the temperature field in various research fields. Most of the temperature field is based on the heat conduction equation to build.

\section{The Models}

Assumptions. We assume that the indoor temperature is 25 degrees Celsius and it won't change during the process of the bath. We assume that the initial water temperature is uniform in bathtub.

We assume that the bathtub converts heat evenly in all directions.

\section{Definitions.}

Table 1 Variables

\begin{tabular}{cll}
\hline Variable & Definition & Units \\
\hline $\mathrm{Q}$ & Heat & $\mathrm{J}$ \\
$\mathrm{K}$ & Heat Transfer Coefficient & $\mathrm{W} /\left(\mathrm{m}^{2} \cdot{ }^{\circ} \mathrm{C}\right)$ \\
$\mathrm{A}$ & The area of heat transfer & $\mathrm{m}^{2}$ \\
$\Delta \mathrm{t}$ & The average temperature difference between the hot and cold fluids & ${ }^{\circ} \mathrm{C}$ \\
$\mathrm{t}_{\mathrm{i}}$ & The temperature of tube & ${ }^{\circ} \mathrm{C}$ \\
$\lambda$ & The level of heat exchange & $\mathrm{W} /\left(\mathrm{m}^{2} \cdot{ }^{\circ} \mathrm{C}\right)$ \\
$\mathrm{r}$ & The level of the disturbance & $\mathrm{NONE}$ \\
$\overline{\mathrm{t}}$ & The average temperature & ${ }^{\circ} \mathrm{C}$ \\
$\mathrm{T}$ & The present water temperature & ${ }^{\circ} \mathrm{C}$ \\
$\mathrm{T}_{0}$ & The initial temperature of the water & ${ }^{\circ} \mathrm{C}$ \\
\hline
\end{tabular}

Blank Bathtub with Nothing but Water. First, we build a simple model of bath through simulating the environment of the bathroom to describe the change of the hot water. In our model, 
we simplify the bathtub as a cube tank, and we define $1500 \mathrm{~mm}$ as its length, $800 \mathrm{~mm}$ as its width and $500 \mathrm{~mm}$ as its height. Now we set the water temperature at $39^{\circ} \mathrm{C}$ as its initial state [1].

From the initial state, the state of the hot water is slowly changed by the constraints and rules that we give. Then how does the water temperature changes?

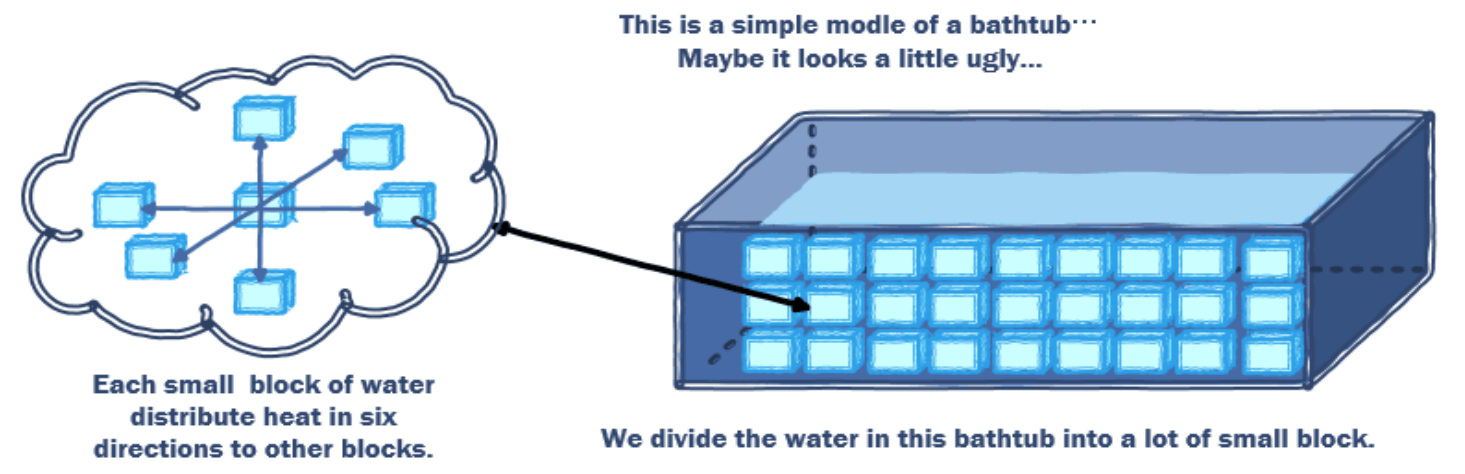

Figure 1.

Please look at the Fig. 1.We put the hot water in the bathtub into a lot of little water cubes. Each of the water cube exchanges heat with the surrounding cube. It seems that we solidify the water.

Actually, we don't mean that. We just make a space division to the bathtub, and each small space represents the state of changes with temperature [2]. And the water wanders between those little water cubes and exchange their heat to influence the temperature distribution of the bathtub.

So how do a little water cube influence the other cubes around itself ?

Process of heat transfer is not made in a single manner, but the combination of two or even three kinds of heat transfer modes.

Heat transfer from the hot fluid to the cold one is usually expressed as Equation 1 [3].

$$
Q=K A \Delta t
$$

It is easy to see, if we change the value of $K, A$ or $\Delta t$, we can control the strength of heat exchange.

Now we only consider how to change parameter K. K has a connection with the flow water. We use a disturbance to simulate water flow. When disturbance increases, the water in the bathtub become more active. So the disturbance represents $K$. Then we build our model by the mode of heat exchange and the flow of water [4]. Here is the Equation 2, we crate for our model:

$$
t_{i}=\lambda r \bar{t}+(1-\lambda r) t_{i}
$$

To simulate the process of heat transfer. We change the range of one little water tube's influence by $r$. The shade of color represent the degree of center tube's influence [5]. If $\mathrm{R}$ become bigger, the influence spread farther.

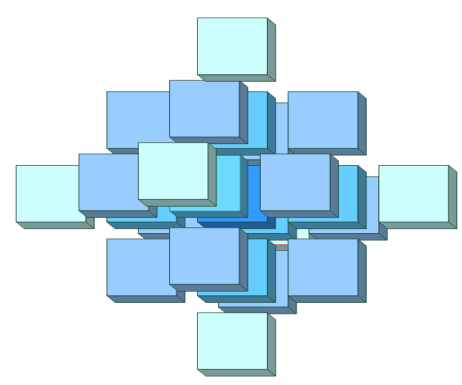

Figure 2.

Here is Fig. 2. We use Matlab to get the temperature field model of the bathtub. 


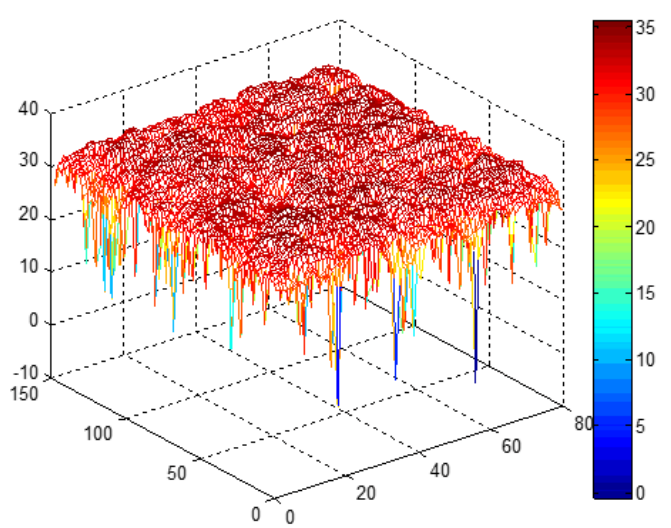

Figure 3. When the $\mathrm{r}$ is small, no one disturbs the hot water

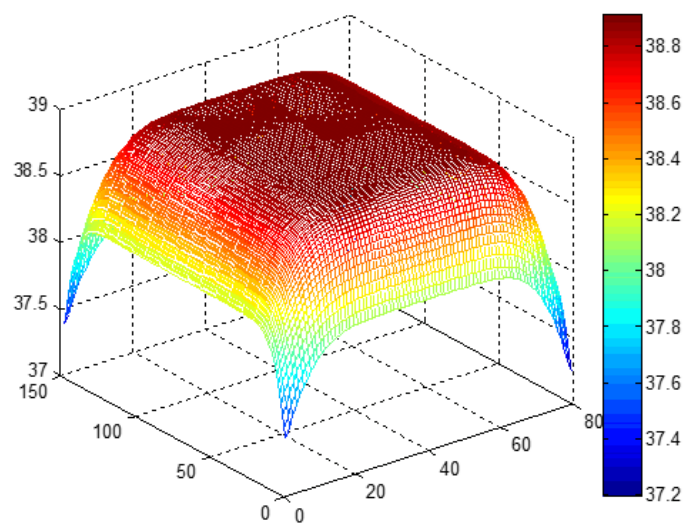

Figure 4. When the $\mathrm{r}$ is big, like someone stirs up the hot water

Fig. 3 and Fig. 4 are the result of simulation. As you can see, when the disturbance increased gradually, the distribution of the temperature field become more uniform. At the same time, the rising $r$ increases the heat exchange of air and water, resulting in a decrease of the water temperature.

Bathtub with One Tried Man. We put the people who want to have a bath into a combination made by two cuboids, and the combination always keeps its temperature at $36.5^{\circ} \mathrm{C}$ [6]. One cuboid represents the upper body of the person, and another represents the lower body. May it is too crude, but it really make our work more easy [7]. Here is Fig. 5.

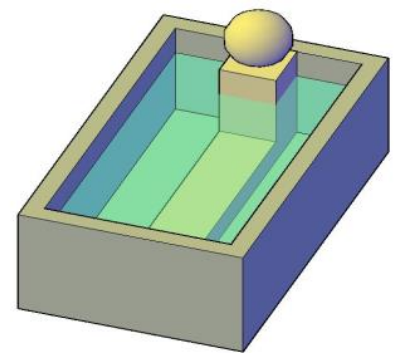

Figure 5. This is a schematic of the bathtub and the tired person who want a hot bath

Fig. 6 and Fig. 7 show the initial state we set for bath models, of course, there is a man in the bathtub.

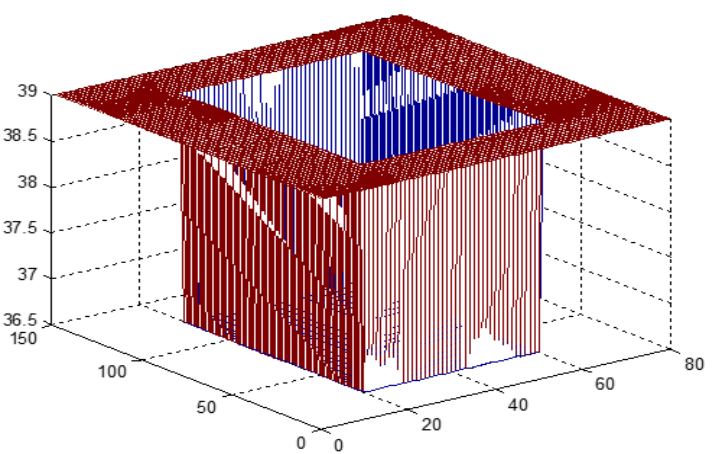

Figure 6. This one represents the distribution of the water temperature in the upper bathtub

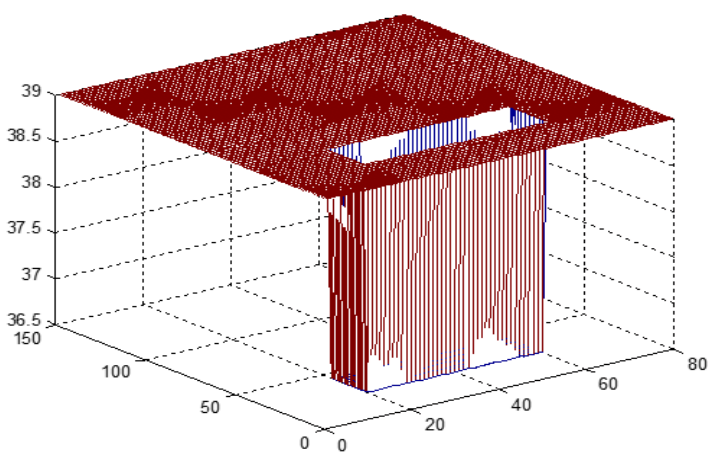

Figure 7. And this one represents the distribution of the water temperature in the lower bathtub 
We all know that the temperature of the water in the tub is higher than the temperature of environment. So the hot water will transfer the heat to the outside, at the same time, it will become colder [8].

The accurate speed and the magnitude of decline of the water temperature ask for a large number of experiments. We don't have condition to do this work and we don't know how to do it [9]. So we get the relation of the water temperature by some documents.

From those documents, we understand that the cooling process of hot water can be expressed as Equation 3 [10].

$$
T=T_{0}+M e^{-\frac{\tau}{V}}
$$

Based on this equation, we get Fig. 8 that the change of temperature field as time passed by.
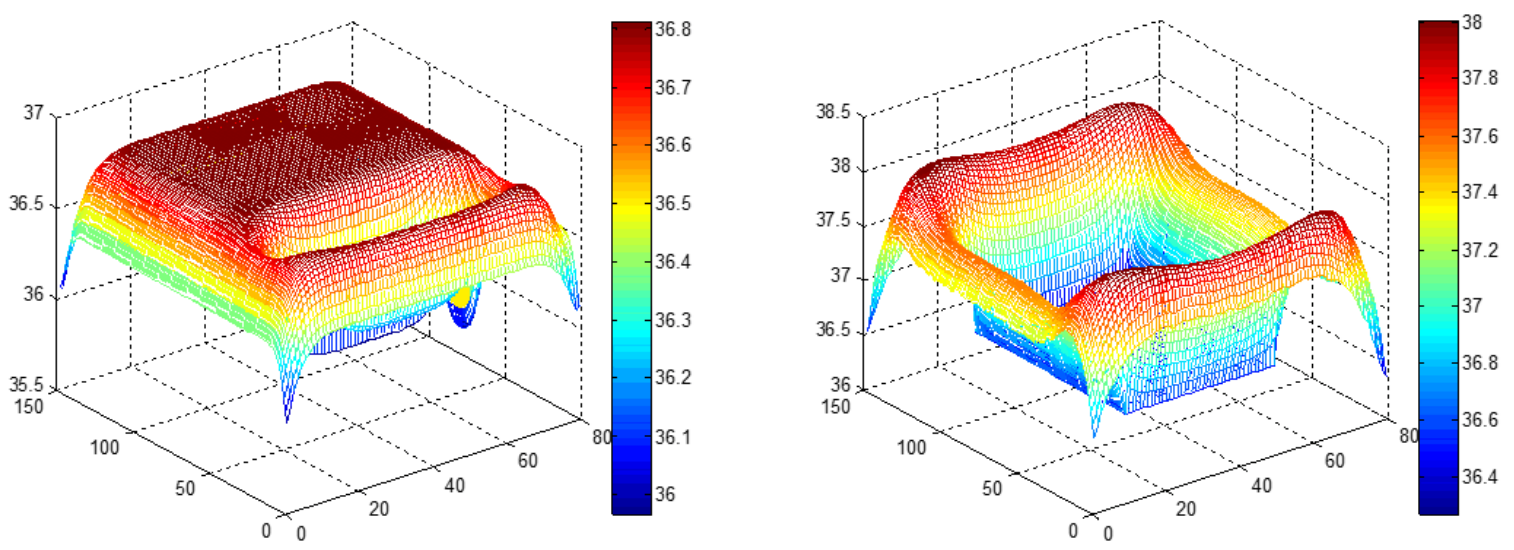

Figure 8 .

\section{Conclusion}

People's actions in the bath crock has a great influence on temperature field. People's move will drive the movement of water. We strengthen the disturbance of water, it in favor of the temperature diffusion. At the same time, the surface of the water flow more quickly. It can make evaporation and heat preservation of the water more quickly. It will go ill with keeping the temperature.

In summary, our strategy is to join the little hot water, at the same time, people take small stir in the water.

\section{Reference}

[1] Zhaorong Yi. The model of water temperature environment of aquiculture [J]. Xiaozuojia xuankan, 2015(5):28-29.

[2] Songfeng Gao, Qianqi Yang. Application of Reservoir Water Temperature3-D Model [J]. Pollution control technology, 2015,28(2): 15-17.

[3] Weiwen huang. The thermal and fluid machinery[M].Beijing: China electric power press,2007.

[4] Ping Hu, Yi Liu, Zhongmin Tang. Numerical prediction of water temperature in reservoir [J]. Shuili xuebao,2010,41(9): 1045-1053.

[5] Johnson, B H. A review of multidimensional reservoir hydrodynamic modeling [J]. American society of civil Engineers. 2010. 
[6] Junqiao Hu, Dequn Li, Yiqiang Hu. Modeling and Simulating of 3-D Transient Temperature Fields in Injection Mould [J]. China plastics, 1995, 9(5): 52-59.

[7] Donghui Li, Qibin Xin, Hui Chen. An Implementation of the Castings'3D Temperature Field Post-Processing Technique on Matlab [J]. Journal of Shenyang in statute of technology, 2001, 20(3): 5-10.

[8] Fox J. F. Intermediate-disturbance hypothesis [J]. science, 1979(4399): 1344-1345.

[9] Yun Deng. Study on the Water Temperature Prediction Model for the Huge and Deep Reservoir [D]. Sichuan: Sichuan university, 2003: 82-83.

[10] Shekede M. D. , Kusangaya S. , Schmidt K. Spatio-temporal variations of aquatic weeds abundance and coverage in Lake Chivero, Zimbabwe [J]. Physics and Chemistry of the Earth, 2008(33): 714-721. 